\title{
Estilo de aprendizaje como parte de la tecnología educativa para el proceso de enseñanza
}

\author{
Learning style as part of educational technology for the \\ teaching process
}

\author{
Andrés Lárez \\ andres_larezc@hotmail.com \\ Código ORCID: 0000-0001-5624-8185 \\ Universidad del Zulia, Venezuela
}

- Artículo recibido en abril 2019

- Arbitrado en mayo 2019

- Publicado en julio 2019

\section{RESUMEN}

El propósito fue analizar el estilo de aprendizaje como parte de la tecnología educativa para el proceso de enseñanza en el Núcleo Costa Oriental del Lago de la Universidad del Zulia. La investigación se enmarcó en un tipo descriptivo, con diseño de campo, no experimental-transversal, tomándose una población de 44 profesores correspondientes al programa ingeniería. La técnica de recolección de datos fue un cuestionario constituido por 9 ítems con respuestas tipo Likert; el cual fue validado a través del juicio de experto y la confiabilidad por el método Alpha Cronbach , arrojando un 0,86. Para el procesamiento de los datos, se utilizó el método de estadística descriptiva donde se pudo constatar que casi siempre el proceso de enseñanza-aprendizaje se lleva a cabo mediante una serie de estilos, que le permita al estudiante observar las experiencias desde diferentes perspectivas para actuar rápidamente con seguridad ante ideas y proyectos que le atraen.

Palabras clave: estilo de aprendizaje; estudiante; experiencias; proceso de enseñanza; tecnología educativa

\section{ABSTRACT}

The purpose was to analyze the learning style as part of the educational technology for the teaching process in the Core Eastern Coast of the Lake of the University of Zulia. The research was framed in a descriptive type, with a field design, not experimental-transversal, taking a population of 44 professors corresponding to the engineering program. The data collection technique was a questionnaire consisting of 9 items with Licket-type responses; which was validated through expert judgment and reliability by the Alpha Cronbach method, yielding 0,86. For the data processing, the descriptive statistical method was used where it was found that almost always the teaching-learning process is carried out through a series of styles, which allows the student to observe the experiences from different perspectives to act quickly with confidence before ideas and projects that appeal to you.

Keywords: learning style; student; experiences; teaching process; educational technology 


\section{INTRODUCGIÓN}

Las universidades en el ámbito mundial están desarrollando constantes cambios para adaptarse a las nuevas condiciones del contexto en el cual se desenvuelven y se orientan cada vez más desde una perspectiva competitiva y que hace que las universidades necesiten mejorar sus procesos tecnológicos para asegurar su permanencia en la sociedad.

Es por ello que en el transcurrir del tiempo el ser humano realiza innovaciones en la vida, especialmente en las universidades para que los procesos sean más operativos, lo cual se está logrando gracias al avance de la tecnología. Esto conduce a la necesidad de adquisición de conocimientos y técnicas que mejoren el desarrollo de las actividades y herramientas que facilitan los procesos de aprendizaje dentro de las universidades.

En este sentido, la UNESCO (1999) en su informe mundial sobre la educación, señala que las nuevas tecnologías constituyen una forma totalmente de educación y ofrecen una compleja serie de oportunidades y tareas a las instituciones de enseñanza de todo el mundo. Por su parte, Tébar (2003) expresa que el entorno del aprendizaje virtual es un programa informático interactivo de carácter educativo que posee una capacidad de comunicación integrada.
Por tanto, los usos reales de los equipos y programas informáticos en el trabajo educacional cotidiano se realizan con el objeto de integrarlos a la práctica educativa, la cual debe efectuarse a la luz de las directrices del nuevo diseño curricular, en el que la tecnología de la información tiene una presencia intensa y fecunda. En términos generales, la tecnología educativa, según Esteves (2008), es el resultado de las aplicaciones de diferentes concepciones y teorías educativas para la resolución de un amplio espectro de problemas $y$ situaciones referidos a la enseñanza y el aprendizaje, apoyadas en las TIC (tecnologías de información y comunicación).

Dicho aspecto permite reflejar mediante un ámbito práctico la premisa desde la cual la tecnología llegó para revolucionar el mundo educativo, haciendo de cada persona receptor de la información, un ser social adentrado en la construcción colectiva del aprendizaje idóneo, tomando elementos externos al aula y convirtiendo éstos últimos en canales de flujo de aprendizaje extensos.

En referencia a lo descrito; el proceso de enseñanza posee poco desarrollo con las grandes transformaciones; a través del enriquecimiento y utilización de la tecnología educativa, lo cual se convierte en un vehículo para el aprendizaje del contenido de las materias y del uso efectivo de las mismas. Sin embargo, la 
premisa es que con un conocimiento básico del uso de una herramienta tecnológica determinada, el estudiante puede utilizarla para desarrollar las diferentes actividades.

Otras de las premisas, es tratar de cambiar las formas tradicionales de enseñanza que hasta el momento se manejan, como lo son en su mayoría: conferencias, clases prácticas, seminarios, donde los aspectos académicos como motivación y comunicación con el estudiante son de un nivel no óptimo para lo deseado por la educación, como las tecnologías que están en un proceso de búsqueda y transformaciones, lo cual es preciso caminar a la par de la nueva era.

De acuerdo a ello, tomando en cuenta que las condiciones actuales de la sociedad exigen seres humanos que posean una mentalidad científica ni dogmática ni esquemática, sin la importancia de cómo producir lo que saben sino que sientan deseos de innovar, que posean una conducta transformadora, donde se considere que los esfuerzos para alcanzar el conocimiento productivo y no reproductivo en función del desarrollo de la sociedad, deben ser aún mayores; ya que no basta con adquirir la información, es preciso buscarla, seleccionar de ella lo necesario para construir nuevos procesos de enseñanza y aprendizaje.
Según Spiegel (2008) varios investigadores, entre los que se incluyen Charles Hoban, James Finn y Edgar Dale, expresan que para adaptarse a las necesidades de la sociedad actual, las instituciones de educación superior deben flexibilizarse para desarrollar vías de integración de las tecnologías educativa en los procesos de formación.

Para ello es de vital importancia considerar como factor importante la idiosincrasia en cada una de las instituciones al integrar la tecnología educativa en los procesos de la enseñanza universitaria, a fin de aplicar una nueva concepción tanto en los estudiantes como en los docentes, puesto que los mismos pasarán a jugar un rol diferente, no desde el punto de vista del concepto estudiantil, sino de las nuevas formas de aprendizaje que tendrán que desarrollarse en la utilización de la tecnología, así como cambios de rol en los profesores, cambios administrativos en relación con los sistemas de comunicación, con el diseño y la distribución de la enseñanza.

Por lo planteado, la enseñanza basada en la tecnología educativa se produce mediante el hecho de involucrar al educando en su aprendizaje constructivo, partiendo de la premisa en la cual al usar herramientas didáctico - tecnológicas, sea él mismo el que se interese por internalizar lo impartido, basados en aquella información dispuesta con creatividad, intencionalidad, claridad, entre otras. 
Sin embargo, el aspecto esencial de la tecnología educativa se centra en la transformación radical de la educación, la relación de la persona con la educación innovadora basada en que al presenciar clases magistrales tradicionales no se expanden las habilidades cognitivas en todo su potencial, pues el estudiante sólo se predispone a escuchar la clase y salir de ella, caso contrario cuando se hacen prácticas didácticas y tecnológicas en la cual se despierte el interés del aprendiz por lo enseñado.

Es pertinente mencionar y aportar información básica sobre los aspectos históricos de la tecnología educativa, en tal sentido, se expone lo planteado por Alonso (2006), quien refiere que la historia de la tecnología educativa sufrió bastantes cambios a lo largo del tiempo, consecuencia de la evolución de la sociedad (que vive una etapa de rápido desarrollo tecnológico) y de los cambios que se producen en las ciencias que la fundamentan.

Además de lo anterior, se centró también en un sentido artefactual (al centrarla en los medios, entendidos únicamente como dispositivos tecnológicos utilizados con fines instructivos) y una clara dependencia de la psicología del aprendizaje, que la situaron en una perspectiva técnicoempírica, los cambios de paradigma en algunas de disciplinas que la sustentaban (psicología del aprendizaje, teoría de la comunicación, sociología) permitieron evolucionar y encontrar nuevos enfoques bajo una perspectiva cognitiva mediacional y crítica.

Por tanto, lo descrito por el autor resulta relevante para inferir que la tecnología educativa evolucionó gradualmente, con la finalidad de centrar su propósito en reflexión y construcción del conocimiento, empleando técnicas, estrategias, métodos y procedimientos aplicables a cualquier sujeto según sus necesidades educativas partiendo desde un enfoque constructivista.

Aunado a lo anteriormente planteado, la tecnología educativa es integradora (de cada ente social - educativo) y viva (transcurre día con día). Ejemplificando lo mencionado, en una indagación cualitativa informal, y tomando como referencia una casa de estudios de la región zuliana ( $\mathrm{La}$ Universidad del Zulia), se detectó en el Núcleo Costa Oriental del Lago, que algunas veces no se hace uso de herramientas tecnológicas integradoras de la participación activa en el aprendizaje formal de la persona a través de modelos de enseñanza aprendizaje.

A su vez, no brinda la posibilidad de vincular los elementos tanto tecnológicos como organizativos dentro del proceso educativo y del docente. Con esto, no se inventan nuevas metodologías para que la utilización de herramientas tecnológicas en la educación abra el camino hacia otras perspectivas respecto a una enseñanza 
mejor, ya que las mismas no se dan dentro de la realidad universitaria en general.

Por otro lado, la relación entre docentes y estudiantes; es una problemática, pues existen comentarios tanto positivos como negativos ante la presencia de las tecnologías en la educación, suscitándose así una serie de riesgos adicionales difícilmente controlables por los docentes $\mathrm{u}$ otros responsables de la educación. Existen deficiencias en la proyección de las clases por parte del profesor para organizar el proceso de enseñanza del estudiantado. A su vez, se no se visualiza un efecto claramente positivo y determinante de las tecnologías educativas en el rendimiento estudiantil, ya que no se encuentra dentro de los niveles aceptables dentro de la institución.

Por ello, se hace de carácter relevante que quienes están a cargo de las universidades tomen conciencia de la importancia de poseer dentro de éstas los medios necesarios para que los docentes actualicen y optimicen la educación juntos a sus estudiantes; ya que en años anteriores, la educación en las institución universitaria objeto de estudio se hacía meramente conductista sin elementos que favorecieran la captación efectiva de la información impartida por los profesores, la participación en la construcción de los saberes no se daba voluntariamente, dicha problemática se intuye que proviene por la ausencia de medios didáctico de enseñanza y recursos tecnológicos.

Sintetizando todo lo descrito, la tecnología educativa se hace para hacer efectivo los procesos educativos, como estrategia integradora, social, constructivista $u$ óptima, realizada mediante medios didácticos - tecnológicos que promuevan la captación de atención y receptividad por parte del estudiante. A su vez, la misma se decreta fundamental, porque se hace para mejorar la calidad de internalización del contenido impartido, originando como consecuencia altos niveles de interés, aprendizaje, creatividad, actitudes críticas, pensamientos reflexivos, entre otras características que acreditan dicha modalidad de enseñanza educativa.

En tal sentido, como forma de minimizar esta problemática lo cual está retrasando el aprendizaje de los estudiantes, se propone un estudio sobre el uso de la tecnología educativa como herramienta para el proceso enseñanza de los mismos, con el fin de hacer del aprendizaje algo agradable y práctico.

Tomando en consideración lo expuesto, se formula la siguiente interrogante: ¿El estilo de aprendizaje permite la integración de la tecnología educativa como herramienta para el proceso de enseñanza en el Núcleo Costa Oriental del Lago de la Universidad del Zulia? 


\section{Tecnología educativa}

Salinas (2004) considera que la tecnología educativa se encarga del estudio, desarrollo, implementación, almacenamiento y distribución de la información mediante la utilización de hardware y software como medio de sistema informático.

Desde esta perspectiva, la tecnología educativa es una parte de las tecnologías emergentes que habitualmente suelen identificarse con la tecnología de la información y comunicación, los cuales hacen referencia a la utilización de medios informáticos para almacenar, procesar y difundir todo tipo de información o procesos de formación educativa. Por su parte; según la Asociación Americana de las Tecnologías de la Información (2000) considera que

Es el estudio, diseño, desarrollo, fomento, mantenimiento $y$ administración de la información por medio de sistemas informáticos, esto incluye todos los sistemas informáticos no solamente la computadora, este es solo un medio más, el más versátil, pero no el único; también los teléfonos celulares, la televisión, la radio, los periódicos digitales, entre otros. (p. 7)

Esas tecnologías se presentan cada vez más como una necesidad en el contexto de la sociedad donde los rápidos cambios, el aumento de los conocimientos y las demandas de una educación de alto nivel constantemente actualizada se convierten en una exigencia permanente.

$\mathrm{Al}$ respecto, Tébar (2003) afirma que la tecnología educativa es aquella herramienta computacional e informática que procesan, almacenan, sintetizan, recuperan y presentan información representada de la más variada forma, las cuales constituyen nuevos soportes $\mathrm{y}$ canales para dar forma, registrar, almacenar y difundir contenidos informacionales.

En referencia a las definiciones anteriores y desde el punto de vista del investigador, se establece que la tecnología educativa tienen como finalidad tratar sobre el empleo de computadoras o aplicaciones informáticas, así como transformar, almacenar, gestionar, proteger, difundir y localizar los datos necesarios para cualquier actividad humana; ya que la instrumentación tecnológica es una prioridad en la comunicación.

Por tanto, la relación entre la tecnología de la información y comunicación con la educación tiene dos vertientes. Por un lado, los ciudadanos se ven abocados a conocer y aprender sobre la primera y por otro, las mismas pueden aplicarse al proceso de enseñanza de cualquier institución de educación universitaria.

\section{Enseñanza}

La esencia de la enseñanza está en la transmisión de información mediante la comunicación directa o apoyada en la 
utilización de medios auxiliares, de mayor o menor grado de complejidad y costo. Para Martín y Jiménez (2003); tiene como objetivo lograr que en los individuos quede, como huella de tales acciones combinadas, un reflejo de la realidad objetiva de su mundo circundante que, en forma de conocimiento del mismo, habilidades y capacidades, lo faculten y, por lo tanto, le permitan enfrentar situaciones nuevas de manera adaptativa, de apropiación y creadora de la situación particular aparecida en su entorno. Establece Valera (2000); que el propósito esencial de la enseñanza es

La transmisión de información mediante la comunicación directa o soportada en medios auxiliares, que presentan un mayor o menor grado de complejidad y costo. Como resultado de su acción, debe quedar una huella en el individuo, un reflejo de la realidad objetiva, del mundo circundante que, en forma de conocimiento, habilidades y capacidades, le permitan enfrentarse a situaciones nuevas con una actitud creadora, adaptativa y de apropiación. (p. 68)

Como consecuencia del proceso de enseñanza ocurren cambios sucesivos e ininterrumpidos en la actividad cognoscitiva del estudiante. Con la ayuda del maestro o profesor, que dirige su actividad conductora $\mathrm{u}$ orientadora hacia el dominio de los conocimientos, así como a la formación de habilidades $y$ hábitos acordes con su concepción científica del mundo, el estudiante adquiere una visión sobre la realidad material y social; ello implica necesariamente una transformación escalonada de la personalidad del estudiante universitario.

En la enseñanza se sintetizan conocimientos. Se va desde el no saber hasta el saber; desde el saber imperfecto, inacabado e insuficiente hasta el saber perfeccionado, suficiente y que, sin llegar a ser del todo perfecto, se acerca a la realidad. La enseñanza se propone reunir los hechos, clasificarlos, compararlos $\mathrm{y}$ descubrir sus regularidades, sus necesarias interdependencias, tanto las de carácter general como las internas.

Cuando se recorre el camino de la enseñanza, al final, como una consecuencia obligada, el neurorreflejo de la realidad habrá cambiado, tendrá características cuantitativas y cualitativas diferentes, no se limitará sólo al plano abstracto sino que continuará elevándose más y más hacia lo concreto intelectual, o lo que es lo mismo, hacia niveles más altos de concretización, donde, sin dejar de considerarse lo teórico, se logra un mayor grado de comprensión del proceso real.

Al respecto, Sancho (2003) expresa que todo proceso de enseñanza científica es un motor impulsor del desarrollo que, consecuentemente, y en un mecanismo de retroalimentación positiva, favorecerá 
su propio progreso en el futuro, en el instante en que las exigencias aparecidas se encuentren en la llamada zona de desarrollo próximo del individuo al que se enseña. Este proceso de enseñanza científica deviene en una poderosa fuerza de desarrollo, que promueve la apropiación del conocimiento necesario para asegurar la transformación continua del entorno del estudiante universitario en aras de su propio beneficio como ente biológico.

Por tanto, la enseñanza existe para el aprendizaje; sin ella, este no se alcanza en la medida y cualidad requeridas; mediante ella, el aprendizaje estimula. Así, estos dos aspectos, integrantes de un mismo proceso, conservan cada uno por separado sus particularidades, al tiempo que conforman una unidad entre la función orientadora del profesor y la actividad del educando. La enseñanza es siempre un complejo proceso dialéctico donde su evolución está condicionada por las contradicciones internas, que constituyen en indetenibles fuerzas motrices de su propio desarrollo, regido por leyes objetivas que hacen posible su concreción.

\section{Aprendizaje}

Considerando el aprendizaje desde planteamientos socio-constructivistas, el mismo encabeza un abanico de actividades que, contemplando su diversidad cognitiva y de intereses, procurarán la máxima autonomía de los estudiantes universitarios en la organización de sus propias experiencias de aprendizaje, el cual puede ser analizado desde distintas perspectivas según las teorías del aprendizaje. Sin embargo, al aprendizaje se le puede considerar, según Gardner y Alexander (1999) como

Un proceso de naturaleza extremadamente compleja caracterizado por la adquisición de un nuevo conocimiento, habilidad o capacidad, debiéndose aclarar que para que tal proceso pueda ser considerado realmente como aprendizaje, en lugar de una simple huella o retención pasajera de la misma, debe ser susceptible de manifestarse en un tiempo futuro y contribuir, además, a la solución de situaciones concretas, incluso diferentes en su esencia a las que motivaron inicialmente el desarrollo del conocimiento, habilidad o capacidad. (p. 15)

El aprendizaje, si bien es un proceso, también resulta un producto por cuanto son, precisamente, los productos los que atestiguan de manera concreta los procesos. Aprender, para algunos, no es más que concretar un proceso activo de construcción que lleva a cabo en su interior el sujeto que aprende (teorías constructivistas). No debe olvidarse que la mente del educando, su sustrato material neuronal, no se comporta solo como un sistema de fotocopiado humano que sólo reproduce en forma mecánica, más o menos exacta y de forma instantánea, los aspectos de la realidad objetiva que se introducen en el referido soporte receptor neuronal. 
Por tanto, el estudiante universitario ante tal influjo del entorno, de la realidad objetiva, no copia simplemente sino también transforma la realidad de lo que refleja, esto indica que construye algo propio y personal con los datos que la antes mencionada realidad objetiva le entrega, debiéndose advertir sobre la posibilidad de que si la forma en que se produce la transmisión de las esencialidades reales resultan interferidas de manera adversa o debido al hecho de que el propio educando no pone, por parte de sí, interés o voluntad, que equivale a decir la atención y concentración necesarias, sólo se alcanzarán aprendizajes frágiles y de corta duración.

Asimismo, en el aprendizaje influye de manera importante el significado que lo que se aprende tiene para el estudiante en cuestión, pudiéndose hacer una distinción entre el llamado significado lógico y el significado psicológico de los aprendizajes. Por muy relevante que sea en sí mismo un contenido de aprendizaje, es necesario que la persona lo trabaje, lo construya $\mathrm{y}$, al mismo tiempo, asigne un determinado grado de significación subjetiva para que se plasme o concrete un aprendizaje significativo es decir que se produzca una real asimilación, adquisición y retención del conocimiento ofrecido.

El aprendizaje, por su esencia $y$ naturaleza, no puede reducirse $y$, mucho menos, explicarse sobre la base de los planteamientos de las llamadas corrientes conductistas o asociacionistas y cognitivas. No puede concebirse como un proceso de simple asociación mecánica entre los estímulos aplicados y las respuestas provocadas por estos, determinadas tan sólo por las condiciones externas imperantes, donde se ignoran todas aquellas intervenciones, realmente mediadoras y moduladoras, de las numerosas variables inherentes a la estructura interna, principalmente del subsistema nervioso central del sujeto cognoscente, que aprende.

A su vez, no es simplemente la conexión entre el estímulo y la respuesta, la respuesta condicionada, el hábito es, además de esto, lo que resulta de la interacción del estudiante que se apropia del conocimiento de determinado aspecto de la realidad objetiva, con su entorno físico, químico, biológico $\mathrm{y}$, de manera particularmente importante con su realidad social.

\section{Estilos de aprendizajes}

El término estilo de aprendizaje hace énfasis en el hecho de que cuando se quiere aprender algo cada facilitador utiliza su propio método o conjunto de estrategias. Aunque las estrategias concretas que se utilizan varían según lo que se desea aprender, cada uno tiende a desarrollar unas preferencias globales. Esas preferencias o tendencias a utilizar más unas determinadas maneras de aprender que otras constituyen lo que es el estilo de aprendizaje. Por tanto, las diferencias entre los estudiantes son 
múltiples: de tipo cultural, intelectual, afectivo. Cada estudiante tiene su estilo de aprendizaje en el que, según Sancho (2003) se pueden clasificar en:

- Las preferencias perceptivas: visual, auditiva.

- Las preferencias de respuesta: escrita, oral, selección entre varias respuestas.

- El ritmo de aprendizaje (el tiempo necesario).

- La persistencia en las actividades y la responsabilidad de la misma.

- La concentración y la facilidad para distraerse.

- La autonomía o necesidad de instrucciones frecuentes.

- Las preferencias en cuanto a agrupamiento: trabajo en parejas, en grupo, con adultos.

- Las preferencias en cuanto a los recursos a utilizar: escribir a mano o con el ordenador, ir a bibliotecas o consultar por Internet, enseñanza presencial o virtual.

- La dominancia cerebral: hemisferio derecho o izquierdo.

- Tendencia impulsiva o reflexiva $y$ tendencia analítica o global.

- Actividades preferidas: memorización, interpretación, argumentación, creación.

Según Alonso y Gallego (2003) se define el estilo de aprendizaje como los rasgos cognitivos, afectivos y fisiológicos que sirven como indicadores relativamente estables de cómo los discentes perciben, interaccionan $y$ responden a sus ambientes de aprendizaje. Siguiendo a Kolb (2003) se identifican cuatro estilos, estos son:

Activo: Toma mucha información, capta novedades, se implican con entusiasmo activamente y sin prejuicios en nuevas experiencias (experiencia concreta, percibir).

Reflexivo: Acumula y analiza mucha información antes de llegar a conclusiones, les gusta considerar las experiencias desde distintos puntos de vista, observar y escuchar a los demás (observación reflexiva, pensar).

Teórico: Analiza, sintetiza y estructura la información, integran los hechos en estructuras coherentes (conceptualización abstracta, planear).

Práctico: Aplica la información, descubren los aspectos positivos de las nuevas ideas y las aplican a la primera oportunidad (experimentación activa, hacer).

\section{Proceso de enseñanza y aprendizaje}

Según Alonso y Gallego (2003) el proceso de enseñanza y aprendizaje es la ciencia que permite estudiar a la persona y su entorno en las distintas etapas de aprendizaje que abarca su vida. A través de sus métodos propios estudia el problema presente vislumbrando las 
potencialidades cognoscitivas, afectivas y sociales para un mejor y sano desenvolvimiento en las actividades que desempeña la persona.

De acuerdo a ello, como proceso, es una variable que interviene en el aprendizaje, el cual no siempre es observable y tiene que ver con las estrategias metodológicas y con la globalización de los resultados, a través de varias corrientes psicológicas que definen el aprendizaje de formas radicalmente diferentes.

Por su parte, Fariñas (2001) considera que es la puesta en práctica de la interdisciplinariedad, educación, psicología, pedagogía, planeación, diseño curricular, actualización y profesionalización docente; con el fin de llevar a cabo un avance cualitativo de la infancia hasta la adultez, geriatría y recursos humanos, incluyente de sujetos especiales.

En la misma medida en que se sea consecuente en la práctica con las consideraciones referidas se podrá llegar a influir sobre la eficiencia y eficacia del proceso de aprendizaje según el modelo de la ruta crítica: la vía más corta, recorrida en el menor tiempo, con los resultados más ricos en cantidad, calidad y duración. Hay quienes consideran que cuando se registran pensamientos en base de determinadas sensaciones, en el primer momento, se detiene en el análisis de los detalles pero que más tarde los mismos resultan ubicados en determinadas locaciones de la mente que, equivale a decir, en diferentes fondos neuronales del subsistema nervioso central interrelacionados funcionalmente, para formar o construir partes de entidades o patrones organizados con determinada significación para el estudiante que aprende.

Establecidas estas definiciones, Trigwell et al. (2006) consideran que los profesores que conciben el aprendizaje como información, piensan la enseñanza como transmisión de la información y enfocan su docencia en base a estrategias centradas en el profesor. Por el contrario, los que entienden el aprendizaje como el desarrollo y cambio en las concepciones de los estudiantes, conciben la enseñanza como la ayuda a los estudiantes a desarrollar y cambiar sus concepciones, y enfocan su docencia en base a estrategias centradas en el estudiante.

Dentro del proceso de enseñanza $y$ aprendizaje es fundamental reconocer que todos los elementos que componen el sistema educativo como el programa curricular, modelo educativo, la vida universitaria, las normas de la institución interactúan y afectan entre sí. Sin embargo, es elemental reconocer al proceso de enseñanza-aprendizaje como aquel que le da valor $y$ sentido al sistema. 
El programa curricular, por ejemplo, es un largo proceso de enseñanzaaprendizaje, compuesto por una multitud de subprocesos a través de cursos, sesiones de clase y la vida misma del estudiante. Por lo tanto, se puede enfocar desde tres perspectivas: estudiante, profesor y cuerpo de conocimientos; entendiendo al conocimiento como el resultado de un aprendizaje por competencias, en donde el estudiante desarrolla habilidades y actitudes a partir de determinados contenidos.

Ferres (2004) establece que el proceso de enseñanza-aprendizaje (PEA) puede concebirse como

un viaje por una carretera muy bien asfaltada para llegar a un lugar determinado. Sin embargo, éste mismo proceso, a la luz de las nuevas tecnologías de la informática y la comunicación, se visualiza como un viaje en nave espacial, con una misión definida pero con muchas rutas de navegación en un espacio sin fronteras. (s.n.)

Por consiguiente, el proceso de enseñanza-aprendizaje se desarrolla en un nuevo espacio, amplio, interconectado y con mayores posibilidades de integración. En este nuevo contexto, analiza de qué manera la visión de la educación plasmada en el modelo educativo y principios pedagógicos se ve enriquecida por todo ello. Este proceso cuenta con ocho principios que orientan a los profesores en una misma dirección y encaminan el proceso para el logro de los perfiles profesionales.

Estos principios le dan características propias al modelo pedagógico (modelo andragógico) de enseñanza-aprendizaje, y son los siguientes: aprendizaje por competencias; aprendizaje activo; aprender a aprender; educación personalizada; facilitación del aprendizaje; valoración de la diversidad; evaluación y retroinformación; aprendizaje interdisciplinario. Por tanto, se acuerdo a lo anteriormente, se comprobó que a través de las nuevas tecnologías de la informática y de la comunicación estos principios adquieren nuevas posibilidades de desarrollo.

Sobre la base de la misión del proceso, que es la de formar líderes íntegros y realizar propuestas innovadoras para impulsar la creación de una nueva realidad, se propuso un modelo educativo con competencias generales que debe desarrollar todo estudiante universitario. Entre estas competencias se tienen: el pensamiento crítico; creatividad; comunicación; ciudadanía; toma de decisiones basada en valores; orientación al logro; espíritu empresarial.

Estas competencias del modelo son un fin y a la vez un medio para el aprendizaje. El profesor debe estar preparado para promover un aprendizaje creativo, crítico, que estimule la comunicación, la sensibilidad social y el espíritu emprendedor. Por otro lado, estas competencias se desarrollan de manera transversal a lo largo del currículo. 


\section{Interacción profesor-conocimiento}

Establece Ferres (2004) que el profesor como diseñador de secuencias de aprendizajes, medios y materiales es un nuevo reto que el profesor esté dispuesto a administrar su propio conocimiento y a compartirlo tanto de manera presencial como virtual. En este universo interconectado por redes, el profesor debe diseñar diversas estrategias, seleccionar medios y materiales que faciliten un aprendizaje significativo.

Todo ello constituye un capital importante tanto para la disciplina como para el conocimiento de la institución. Producto de la exploración y la aventura intelectual, el profesor tiene a su disposición un nutrido árbol de conocimientos, que le permite producir múltiples materiales y medios. Es tarea del profesor realizar un diseño contextualizado de guías metodológicas. Para tal diseño debe considerar las características del alumno, sus estilos de aprendizaje y su conocimiento previo. Así como es crucial que el profesor identifique la contribución de su curso para el desarrollo de las competencias profesionales y generales.

\section{Interacción conocimientos-estudiante}

Un problema de la educación es la existencia entre el capital intelectual del profesor, del alumno, de la institución y el entorno. Por tanto se necesita formar una comunidad de aprendizaje que valore el conocimiento y lo integre a través de centros virtuales, a manera de depósitos gestionables de recursos para el aprendizaje. Cambiar de un concepto de la información a redes con fluido de ida y vuelta. De una simple exhibición a una apreciación, crítica y gestión de la información.

De un concepto pasivo de recepción y participación uniforme a un concepto de intervención y personalización. Aquel que participe en una comunidad de aprendizaje virtual tiene a través de estos medios, mayores oportunidades para desarrollar de manera personalizada sus focos de interés y de estudio. Un estudiante autónomo aprovechará este espacio. La consabida fuga del conocimiento no sistematizado se termina con este tipo de comunidades virtuales, cuyo fin principal es publicar para intercambiar y enriquecer el conocimiento.

\section{Interacción profesor-estudiante}

Con mayor razón se necesita una educación personalizada en la que se establezca una relación cercana entre el docente y el alumno, que vaya más allá del horario de una sección. Necesita un profesor convencido de la necesidad de una enseñanza personalizada, en la que se requiere establecer un vínculo afectivo que refleje un sincero interés por el aprendizaje del alumno.

En este diálogo presencial o virtual, el profesor debe saber interrogar, formular preguntas legítimas que exijan al 
alumno que elabore hipótesis, que investigue y sustente sus respuestas. Para el logro de dicha tarea el profesor y el estudiante cuentan con dos espacios: uno presencial y otro no presencial o de aula virtual a través de herramientas de comunicación y colaboración como el foro, el chat, el tablón de anuncios, las evaluaciones, entre otros.

\section{MÉTODO}

La investigación se enmarcó en un tipo descriptivo, con diseño de campo, no experimental-transversal. Se determinó una población constituida por 44 profesores correspondientes al Núcleo
Costa Oriental del Lago de la Universidad del Zulia específicamente del programa de Ingeniería.

La técnica de recolección de datos fue un cuestionario constituido por 9 ítems con posibilidades de respuestas tipo Likert. El cual fue validado a través de la técnica del juicio de experto y la confiabilidad a través del método Alpha Cronbach arrojando un valor de 0,86. Para el procesamiento de los datos, se utilizó el método de estadística descriptiva, a través de la media aritmética, para su interpretación fue diseñado un baremo a través de una tabla de rango, intervalo y categoría, la cual se muestra en la Tabla 1.

Tabla 1. Baremo de análisis para la interpretación del promedio

\begin{tabular}{|c|c|c|c|}
\hline Rango & Intervalo & Categoría & Descripción \\
\hline 1 & $0.00-1.00$ & Bajo Nivel & $\begin{array}{l}\text { Expresa un bajo nivel de compromiso de la } \\
\text { tecnología educativa como herramienta para el } \\
\text { proceso de enseñanza }\end{array}$ \\
\hline 2 & $1.01-2.00$ & Moderado Nivel & $\begin{array}{l}\text { Indica un moderado nivel de la tecnología educativa } \\
\text { como herramienta para el proceso de enseñanza }\end{array}$ \\
\hline 3 & $2.01-3.00$ & Alto Nivel & $\begin{array}{l}\text { Indica la existencia de un alto nivel de compromiso } \\
\text { de la tecnología educativa como herramienta para el } \\
\text { proceso de enseñanza }\end{array}$ \\
\hline 4 & $3.01-4.00$ & Muy Alto Nivel & $\begin{array}{l}\text { Indica un muy alto nivel de compromiso de la } \\
\text { tecnología educativa como herramienta para el } \\
\text { proceso de enseñanza }\end{array}$ \\
\hline
\end{tabular}

\section{RESULTADOS}

En la Tabla 1 se evidencia los resultados para la dimensión estilos de aprendizaje, la cual arrojó un promedio de 2.19, ubicándola en la categoría alto nivel de que el proceso de enseñanza-aprendizaje se lleve a cabo mediante una serie de estilos, que le permita al estudiante observar las experiencias desde diferentes perspectivas, con el fin de actuar rápidamente con seguridad ante ideas $\mathrm{y}$ proyectos que le atraen. 
Tabla 2. Dimensión: Estilos de aprendizaje

\begin{tabular}{ll}
\hline Parámetros Indicadores & Promedios \\
\hline Reflexivo & 2.04 \\
Teórico & 2.17 \\
Práctico & 2.38 \\
Promedios & 2.19 \\
\hline
\end{tabular}

Los resultados mostrados validan lo expuesto por Alonso y Gallego (2003), quienes expresan que en los estilos de aprendizajes los rasgos cognitivos, afectivos y fisiológicos que sirven como indicadores relativamente estables de cómo los discentes perciben, interaccionan y responden a sus ambientes de aprendizaje. Así las cosas, los datos arrojados en la tabla, se evidencian en cada uno de los siguientes indicadores:

Reflexivo. Arrojó un promedio de 2.04, ubicándose en la categoría de alto nivel para que el participante anteponga la reflexión a la acción y observe con detenimiento las distintas experiencias; a su vez, obtener datos para analizarlos con detenimiento antes de llegar a alguna conclusión. De acuerdo a ello, Kolb (2003) indica que el estilo reflexivo acumula y analiza mucha información antes de llegar a conclusiones, les gusta considerar las experiencias desde distintos puntos de vista, observar $y$ escuchar a los demás (observación reflexiva, pensar).

Teórico. Se observa un promedio de 2.17, lo cual indica alto nivel de que el estudiante se integre a la experiencia en un marco teórico de referencia, a su vez; sea persistente en su sistema de pensamiento a la hora de establecer principios, teorías y modelo. $\mathrm{Al}$ respecto, Kolb (2003) indica que el estilo teórico analiza, sintetiza y estructura la información, integran los hechos en estructuras coherentes (conceptualización abstracta, planear).

Práctico. Obtuvo un promedio de 2.38, ubicándose en la categoría de alto nivel para que el estudiante descubra el aspecto positivo de las nuevas ideas para experimentarlas. Por otra parte, ser impaciente cuando hay personas que teorizan dentro de las aulas de clases. Ante este resultado, Kolb (2003) expresa que el estilo práctico aplica la información; descubren los aspectos positivos de las nuevas ideas y las aplican a la primera oportunidad (experimentación activa, hacer).

Tomando en consideración los datos anteriormente descritos, permite establecer que existen preferentes perceptivas para lograr la tecnología educativa como lo son: visual, auditiva; preferencias de respuesta: escrita, oral, selección entre varias respuestas; preferencias en cuanto a agrupamiento: trabajo individual, en parejas, en grupo, con adultos; y 
preferencias en cuanto a los recursos a utilizar, con la finalidad de escribir a mano o con el ordenador, ir a bibliotecas, consultar por Internet, enseñanza presencial o virtual del estudiante en cualquier institución universitaria.

\section{CONCLUSIONES}

Una vez analizado el estilo de aprendizaje como parte de la tecnología educativa para el proceso de enseñanza en el Núcleo Costa Oriental del Lago de la Universidad del Zulia, se afina que casi siempre el proceso de enseñanzaaprendizaje se lleva a cabo mediante una serie de estilos, que le permite al estudiante observar las experiencias desde diferentes perspectivas para actuar rápidamente con seguridad ante ideas $\mathrm{y}$ proyectos que le atraen. Esto se observa en los siguientes indicadores:

Reflexivo: casi siempre el participante antepone la reflexión a la acción y observa con detenimiento las distintas experiencias para obtener datos para analizarlos con detenimiento antes de llegar a alguna conclusión.

Teórico: casi siempre el estudiante integra la experiencia en un marco teórico de referencia, a su vez; sea profundo en su sistema de pensamiento a la hora de establecer principios, teorías y modelo.
Práctico: casi siempre el estudiante descubra el aspecto positivo de las nuevas ideas para experimentarlas, por otra parte, ser impaciente cuando hay personas que teorizan dentro de las aulas de clases.

\section{REFERENGIAS}

Alonso, C. y Gallego, D. (2003). Ley de calidad. Tecnologías de la Información y la Comunicación. Revista de Educación MECD, Diciembre 2002

Alonso, C. (2006).Documento estratégico para la Innovación en la Educación Superior. México

Asociación Americana de las Tecnologías de la Información (Information Technology Association of America. ITAA. (2000)

Esteves, J. (2008). La tercera revolución educativa. Barcelona: Paidós

Fariñas, J. (2001).Psicología Educativa. Selección de lecturas. La Habana, Cuba: Editorial "Félix Varela"

Ferres, J. (2004). Educar en una cultura del espectáculo. Barcelona: Paidós

Gardner, R. y Alexander, P. (1999). Metacognition answered and unanswered. Psychologist Educational

Kolb, A. (2003). Los medios en el momento interactivo de la enseñanza. Barcelona: Editorial Espiral

Martín, A y Jimenez, F. (2003). La formación del profesorado en una sociedad tecnológica., Madrid: Cincel

Salinas, J. (2004). Nuevos ambientes de aprendizaje para una sociedad de la información. Revista Pensamiento Educativo, 20. Pontificia Universidad Católica de Chile 
Sancho, J. (2003). En busca de respuestas para las necesidades educativas de la sociedad actual. Una perspectiva transdisciplinar de la tecnología. Revista Fuentes, № 4

Spiegel, A (2008). Planificando clases interesante. Itinerario para combinar recursos didácticos. Vol. 1. 3era Edición. Recuperado de https:books.google.cl/books?id=jsv99h gMC\&printsec $=$ frontcover $\&$ source $=\mathrm{g}$ bs_ge_summary_r\&cad $=0 \# v=$ onepage \&q\&f=false

Tébar, L. (2003) El perfil del profesor mediador. Madrid: Aula XXI/Santillana
Trigwell, K., Prosser, M., \& Waterhouse, F. (1999). Relations between teachers' approaches to teaching and students' approaches to learning. Higher education, 37(1), 57-70

UNESCO (1999). Declaración Mundial sobre la Educación Superior en el Siglo XXI. Visión y Acción. París

Valera, A. (2000) Las corrientes de la psicología contemporánea. Revisión crítica desde sus orígenes hasta la actualidad. Colombia: Universidad Autónoma de Colombia 